\title{
First trimester fetal echocardiography limitations and its expected clinical values
}

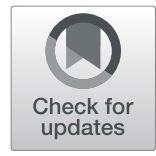

Heba Kamel ${ }^{1 *}$ and Amr Yehia ${ }^{2}$

\begin{abstract}
Background: Fetal cardiac activity could be observed between 6th and 7th gestational weeks, early performance of fetal echocardiography could be implemented to screen for fetal heart disease. The effectiveness of early first trimester fetal echocardiography has not been adequately investigated, especially with modern sonographic technological advances. The purpose of the study is to evaluate the capability to visualize fetal cardiac structures within the first trimester as early as 10th gestational weeks and to elucidate the value of using color Doppler in visualization of cardiac structures within early gestation. A prospective clinical trial conducted on 150 study subjects, 44 of them were twin gestations. Cases were fully assessed by fetal echocardiographic examination from 10th gestational week to 13 gestational weeks in a sequential manner weekly. The research study was conducted at cardiology department fetal unit in one of the tertiary hospitals.

Results: Four chamber view was mostly visualized from 12 gestational weeks, whereas cardiac axis was fully visualized in all cases from 12 gestational weeks; on the other hand, IVC assessment by 2D was satisfactorily visualized in $78.26 \%$ of cases and by color Doppler in $82.61 \%$ of cases at 13 gestational weeks, pulmonary veins were visualized in $21.74 \%$ of cases by $2 \mathrm{D}$ and $43.5 \%$ of cases by color Doppler at 13 gestational weeks, and interestingly, ventricular inflows were satisfactorily visualized in almost all cases from 10th gestational weeks.

Conclusions: First trimester fetal echo is an outstanding enhancement in management pathways of cases susceptible to have fetal cardiac abnormalities permitting early detection of structural cardiac anomalies triggering a cascade of scanning for extra cardiac anomalies to aid in evaluation and assessment of the best management course for those affected cases.
\end{abstract}

Keywords: Fetal echocardiography, Color Doppler, 2D

\section{Background}

Fetal echocardiographic scanning has shown recent great advances in which it was routinely practiced from 20 to 24 gestational weeks to determine the presence of congenital heart diseases [1, 2].

However recently, there is a growing research interest to develop an earlier approach to diagnose major structural cardiac anomalies at earlier gestations within the first trimester that is aided by the upgrading in the frequency of sonographic waves produced from abdominal and vaginal probes and other image enhancing features

\footnotetext{
* Correspondence: heba_kamel123@hotmail.com

${ }^{1}$ Congenital and Structural Heart Disease Unit, Department of Cardiology, Faculty of Medicine, Ain Shams University Hospital, Abbassya, Cairo, Egypt Full list of author information is available at the end of the article
}

integrated to display the fetal structures in a detailed manner as early as 8 gestational weeks [3, 4].

Congenital heart diseases are considered challenging cases as they require multidisciplinary management approach by integrated efforts between the sonographer, obstetrician, and the neonatologists; therefore, early diagnosis could further enhance and improve clinical outcomes by permitting establishment of suitable and safe circumstances during delivery $[5,6]$.

Transposition of great arteries and hypoplastic chambers among other cardiac anomalies are responsible for chief mortalities among neonates particularly when diagnosed after delivery; therefore, the art of fetal echocardiography and systematic scanning of the fetal developing cardiac structures with evaluation of blood flow indices 
and parameters is considered a lifesaving practice in which it permits to secure the affected fetuses to be delivered in the presence of proper NICU capabilities and permits the cardiologist to evaluate and manage the cardiac function to sufficiently prescribe the best and safest management protocol for neonates affected by cardiac disease. Even in cases that could be corrected by cardiac surgery, early intrauterine diagnosis could allow early preparation for life saving surgical approaches $[7,8]$.

However, it is still a developing practice to perform fetal echocardiography within first trimester and it is expected to be a commonly performed approach by enhancements available for fetal imaging among which nuchal translucency is routinely performed in first trimester scanning $[7,9]$.

As research efforts within this field of first trimester scanning increase, that would aid the experience and skills available to rise allowing enhanced safe clinical practice aiding in reducing mortalities among neonates $[10,11]$.

\section{Aim of the work}

The aim of this study is to evaluate the capability to visualize fetal cardiac structures within the first trimester from as early as 10th gestational weeks and to elucidate the value of using color Doppler in visualization of cardiac structures within early gestation.

\section{Methods}

A prospective clinical trial conducted on 150 study subjects, 44 of them were twin gestations in which they were fully assessed by fetal echocardiographic examination from the 10th gestational week to 13 gestational week in a sequential manner weekly, then fetal echo was repeated at the 18th gestational week to compare and contrast the obtained results to those obtained at 10th till 13th gestational weeks. The research study was conducted at cardiology department fetal unit in one of the tertiary hospitals.

The sonographic machines used were GE Vivid E9 and GE vivid s5. Transabdominal scanning, either a $4.5-\mathrm{MHz}$ phased array transducer (M5S) or a 7-MHz phased array transducer (6S) were used. Gestational ages have been obtained using the LMP or early sonographic scan if available. Crown-rump length (CRL) was attained from all recruited study subjects.

\section{Fetal echocardiographic methodology}

Using the transabdominal imaging, the transducer has been situated just above the symphysis pubis, plain of imaging was oriented in a manner to be behind the pubic bone, the gain was adjusted to be most of the time of examination to be above $80 \mathrm{~Hz}$. For visualization of the fetal cardiac anatomical structures (e.g., four chambers, great vessels, and arches) four-chamber imaging, involving the mitral and tricuspid valves, both great arteries with display of great vessels crossing, aortic and ductal arches, and IVC and pulmonary vein, have been approached in all fetuses. The three-vessel imaging permitted assessment of arch position, size, and patency besides relationship to the trachea. Sagittal plane evaluation of the arches supplied valuable sonographic data and findings as regards the arch size and flow.

Furthermore, 2D and color Doppler imaging have been conducted in all research study subjects; both thermal index (TI) and mechanical index (MI) were recorded.

\section{Statistical analysis}

Data were collected, revised, coded, and entered to the Statistical Package for Social Science (IBM SPSS) version 23. Qualitative variables were presented as number and percentages, and comparison between groups regarding qualitative data was done by using chi-square test and/or Fisher exact test when the expected count in any cell found less than 5 . The confidence interval was set to $95 \%$ and the margin of error accepted was set to $5 \%$. So, the $p$ value was considered significant at the level of $<0.05$.

\section{Results}

Table 1 reveals and displays that the mean maternal age was $+/-\mathrm{SD}=26.7 \pm 5.6$ years, mean maternal weight was $73.54+/-12.36$, and mean body mass index (BMI) was $24.63+/-4.75$; gestational age at study entry was mean $\pm \mathrm{SD}=9.5 \pm 0.4$ gestational weeks, as regards parity, 50 cases were PG representing $33.3 \%$ of cases, 75 cases were P1 representing $50.0 \%$ of cases, and 25 cases were $\mathrm{P} 2$ representing 16.7\%; number of singleton gestations $=106$ fetuses representing 70.7\% of total number of fetuses, number of twins $=44$ fetuses representing $29.3 \%$ of fetuses, and total number of fetuses $=194$.

Table 2 reveals and displays fetal echocardiographic findings observed at different gestational ages investigated

Table 1 Demographic data of the studied cases

\begin{tabular}{ll}
\hline & No. $=150$ \\
\hline Maternal age, mean \pm SD & $26.7 \pm 5.6$ \\
GA at study entry, mean \pm SD & $9.5 \pm 0.4$ \\
Weight, mean+/-SD & $73.54+/-12.36$ \\
Body mass index, mean+/-SD & $24.63+/-4.75$ \\
Parity & \\
PG & $50(33.3 \%)$ \\
1 & $75(50.0 \%)$ \\
2 & $25(16.7 \%)$ \\
Number of babies & \\
$\quad$ Single & $106(70.7 \%)$ \\
Twin & $44(29.3 \%)$ \\
Total number of babies & $194(100.0 \%)$ \\
\hline
\end{tabular}


Table 2 Fetal echo cardiac structures observed by echocardiography at different gestational ages investigated by 2D

\begin{tabular}{|c|c|c|c|c|c|}
\hline & \multicolumn{5}{|c|}{ Gestational age (weeks) } \\
\hline & 10th & 11 th & 12th & 13th & 18th \\
\hline Four chamber view & $184(94.8 \%)$ & $188(96.9 \%)$ & $194(100.0 \%)$ & $194(100.0 \%)$ & $194(100.0 \%)$ \\
\hline Cardiac axis & $144(74.2 \%)$ & 186 (95.9\%) & $194(100.0 \%)$ & $194(100.0 \%)$ & $194(100.0 \%)$ \\
\hline Ventricular inflow & $189(97.4 \%)$ & $192(99.0 \%)$ & 194 (100.0\%) & $194(100.0 \%)$ & $194(100.0 \%)$ \\
\hline $\mathrm{Tl}$ & $0.29 \pm 0.1$ & $0.31 \pm 0.11$ & $0.23 \pm 0.09$ & $0.22 \pm 0.10$ & $0.25 \pm 0.1$ \\
\hline Ml & $0.87 \pm 0.15$ & $0.92 \pm 0.21$ & $1.1 \pm 0.27$ & $1.2 \pm 0.28$ & $0.7 \pm 0.2$ \\
\hline Average ultrasound time & $22.5 \pm 12.27$ & $29.3 \pm 10.27$ & $28.7 \pm 9.45$ & $31.2 \pm 9.25$ & $32.4 \pm 8.75$ \\
\hline
\end{tabular}

by $2 \mathrm{D}$; four chamber view was observed in 184 (94.8\%) fetuses at 10th gestational weeks, in 188 fetuses at 11th gestational weeks (96.9\%), at 12th, 13th, 18th gestational weeks in 194 fetuses (100.0\%) (Fig. 1). Cardiac axis was observed in 144 fetuses $(74.2 \%)$ at 10th gestational weeks, 186 fetuses $(95.9 \%)$ at 11 th gestational weeks, 194 fetuses $(100.0 \%)$ at 12 th,13th ,18th gestational weeks. Ventricular inflows were observed 189 fetuses (97.4\%) at 10th gestational weeks, 192 fetuses (99.0\%) at 11th gestational weeks, 194 fetuses $(100.0 \%)$ at 12th, 13th, 18th gestational weeks (Fig. 2). Thermal index at 10th, 11th, 12th, 13th, 18 th gestational weeks $=0.29 \pm 0.1,0.31 \pm 0.11,0.23 \pm$ $0.09,0.22 \pm 0.10,0.25 \pm 0.1$ consecutively. Mechanical index at 10th, 11th, 12th, 13th, 18th gestational weeks = $0.87 \pm 0.15,0.92 \pm 0.21,1.1 \pm 0.27,1.2 \pm 0.28,0.7 \pm 0.2$ consecutively. Average ultrasound time (min) at 10th, 11 th, 12th, 13th, 18th gestational weeks $=22.5 \pm 12.27$, $29.3 \pm 10.27,28.7 \pm 9.45,31.2 \pm 9.25,32.4 \pm 8.75 \mathrm{~min}$ consecutively.
Table 3 reveals and displays fetal echocardiographic findings observed at different gestational ages investigated by $2 \mathrm{D}$ and color Doppler; IVC was observed by 2D in 25 fetuses (12.9\%) at 10th gestational weeks, $87(44.8 \%)$ at 11th gestational weeks, 91 fetuses (46.9\%) at 12th gestational weeks, 155 fetuses (79.9\%) at 13th gestational weeks, and 179 fetuses $(92.3 \%)$ at 18 th gestational weeks, whereas IVC was observed by color Doppler in 171 fetuses $(88.1 \%)$ at 10th gestational weeks, 179 fetuses $(92.3 \%)$ at 11th gestational weeks, 180 fetuses $(92.8 \%)$ at 12th gestational weeks, 182 fetuses (93.8\%) at 13th gestational weeks, and 190 fetuses (97.9\%) at 18th gestational weeks (Fig. 3). Color Doppler in comparison to $2 \mathrm{D}$ in observation of IVC, pulmonary veins, both left and right outflow tracts, crossing of great arteries, aortic arch (Fig. 4) and ductal arch was statistically significant and more superior in detectability by $2 \mathrm{D}$ since most $p$ values $<0.001$. At 13th gestational weeks, color Doppler did not show statistically significant difference in comparison to performance of echocardiography at 18 th gestational weeks (Table 3 ).

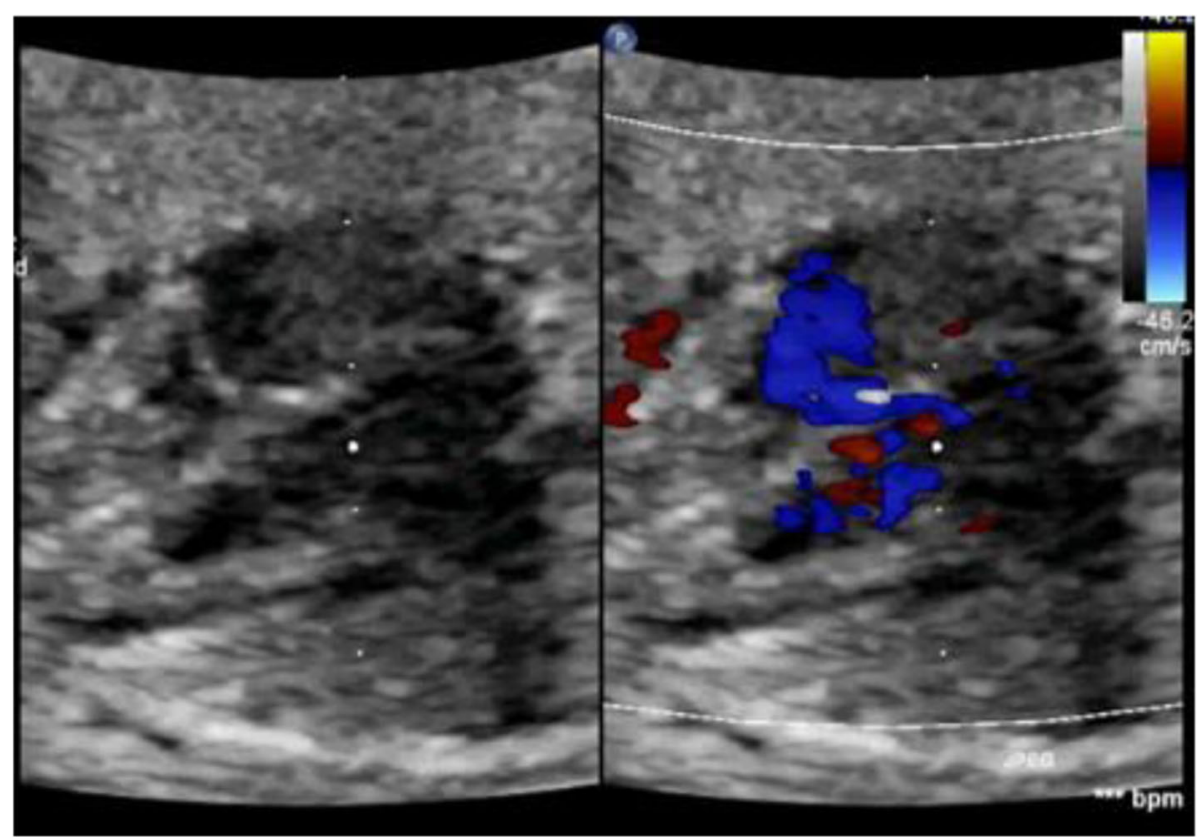

Fig. 1 Four chamber view both by 2D and color Doppler imaging in a case 12-week gestation 


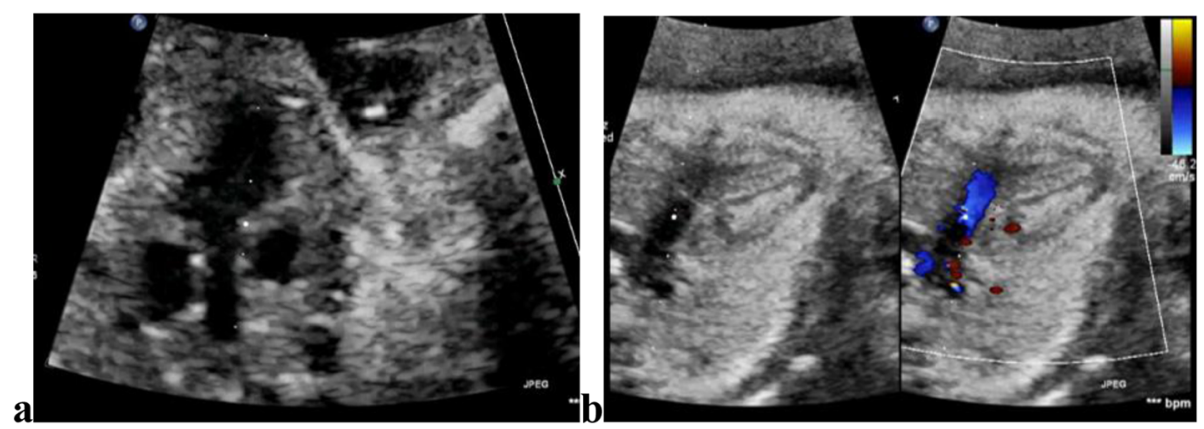

Fig. 2 a Shows left ventricle outflow tract view by 2D imaging in a case 13-week gestation. b Shows right ventricle outflow tract by both 2D and color Doppler imaging in a case 11-week gestation

\section{Discussion}

First trimester echocardiographic screening is an increasingly common practice performed by skilled professionals; however, it requires more skills and experience to be acquired since the degree of visualization of different cardiac structures vary in a considerable fashion and rely on operator and machine related factors. First trimester screening for cardiac anomalies could be used as an adjunct to routine anomaly scanning performed from 18 to 22 gestational weeks $[1,2]$.

Fetal cardiac development could be affected by chronic medical disorders such as DM and could be observed more frequently in families with history of consanguinity and congenital heart disease. So, there is an increasing trend as regards the indications for early first trimester fetal echocardiography aided with advanced technology $[13,15]$.

A cornerstone issue that allows the parents to gain benefit from early diagnosis is to have time to make informed decisions concerning their pregnancy [16].

Furthermore, congenital defects observed within the first gestational trimester could be assessed for their developmental progress and possible prognosis that could be implemented in family discussion and counseling that denotes that more minor fetal cardiac anomalies diagnosed early could be followed up throughout gestation to determine the degree of progression providing clinical opportunities for earlier interventional management and provides a clue for the prognosis post-natal period $[1,3]$.

The current research study findings have interestingly revealed that color Doppler in comparison to $2 \mathrm{D}$ in observation of IVC, pulmonary veins, left and right outflow tracts, crossing of great arteries, aortic arch, ductal arch was statistically significant and more superior in detectability since most $p$ values $<0.001$. Denoting the privilege of color Doppler in detectability of fetal cardiac vessels at early gestational ages that permits detailed fetal cardiac scanning and increases the detectability of cardiac vascular anomalies. That was in agreement with other research group that showed improvement in detection rate with color
Doppler than with 2D imaging for different structures as for example the IVC which was visualized by $2 \mathrm{D}$ imaging in only $4 \%$ in the eighth week, increasing to $13 \%$ by the 10 th week and $80 \%$ by the 13 th week where $\mathrm{CD}$ improved visualization of the inferior vena cava at earlier GAs to $>80 \%$ from 10 weeks [9]. Also, Wener et al. showed that visualization of the $4 \mathrm{CV}$, outflow tract views, and three-vessel view was possible in $90 \%$ of fetuses at 12 to 14 weeks [14]. In our study at 13th gestational weeks, color Doppler did not show statistically significant difference in comparison to performance of echocardiography at 18th gestational weeks. Those findings denote that most severe congenital heart anomalies and structural flow abnormalities are feasible to detect at 13th gestational weeks permitting the obstetrician and pediatric cardiologist to counsel the case about the prognosis and progress of any observed malformation.

Prior research studies more than a decade ago have revealed and displayed that full echocardiographic examination of fetal heart could be performed at satisfactory level at 10th gestational weeks in most cases; however, a prior research study similar to the current research in approach and methodology have shown that color Doppler have a cornerstone value in elucidating the detailed fetal cardiac anatomy especially outflow tracts and venous systems from 10th to 14th gestational weeks; those research findings show great harmony and similarity to the current research study findings. Privilege of early sonographic diagnosis of fetal cardiac pathology also could be a trigger for detailed anomaly scanning for possible associated structural and chromosomal abnormalities [2, 4].

Prior research studies like the current research have mentioned that the best timing for fetal heart evaluation in a complete manner within the first trimester is between $12+0$ and $13+6$ gestational weeks [7].

Hutchinson and co-researchers verified the fact that to obtain a high rate of success for fetal anatomic cardiac evaluation, early fetal echo must be conducted 
Table 3 Fetal cardiac structures observed and investigated by echocardiographic examination using 2D and color Doppler at various gestational ages

\begin{tabular}{|c|c|c|c|c|c|}
\hline & $2 \mathrm{D}$ & $p$ value ${ }^{*}$ & Color Doppler & $p$ value ${ }^{*}$ & $p$ value $^{\ddagger}$ \\
\hline \multicolumn{6}{|l|}{ IVC } \\
\hline 10th w & 25 (12.9\%) & $<0.001$ & 171 (88.1\%) & $<0.001$ & $<0.001$ \\
\hline 11th w & 87 (44.8\%) & $<0.001$ & 179 (92.3\%) & 0.010 & $<0.001$ \\
\hline 12th w & 91 (46.9\%) & $<0.001$ & $180(92.8 \%)$ & 0.016 & $<0.001$ \\
\hline 13th w & 155 (79.9\%) & $<0.001$ & $182(93.8 \%)$ & 0.041 & $<0.001$ \\
\hline 18th w & 179 (92.3\%) & - & 190 (97.9\%) & - & 0.009 \\
\hline \multicolumn{6}{|c|}{ Pulmonary veins } \\
\hline 10th w & $0(0.0 \%)$ & $<0.001$ & $0(0.0 \%)$ & $<0.001$ & 1.000 \\
\hline 11 th w & $0(0.0 \%)$ & $<0.001$ & $6(3.1 \%)$ & $<0.001$ & 0.013 \\
\hline 12th w & $6(3.1 \%)$ & $<0.001$ & $18(9.3 \%)$ & $<0.001$ & 0.011 \\
\hline 13th w & $49(25.3 \%)$ & 0.306 & 89 (45.9\%) & 0.025 & $<0.001$ \\
\hline 18th w & $58(29.9 \%)$ & - & $111(57.2 \%)$ & - & $<0.001$ \\
\hline \multicolumn{6}{|c|}{ Both left and right outflow tracts } \\
\hline 10th w & $45(23.2 \%)$ & $<0.001$ & $128(66.0 \%)$ & $<0.001$ & $<0.001$ \\
\hline 11th w & $146(75.3 \%)$ & $<0.001$ & $179(92.3 \%)$ & $<0.001$ & $<0.001$ \\
\hline 12th w & $150(77.3 \%)$ & $<0.001$ & $185(95.4 \%)$ & 0.004 & $<0.001$ \\
\hline 13th w & $186(95.9 \%)$ & 0.004 & $191(98.5 \%)$ & 0.652 & 0.126 \\
\hline 18th w & $194(100.0 \%)$ & - & $194(100.0 \%)$ & - & 1.000 \\
\hline \multicolumn{6}{|c|}{ Crossing greater arteries } \\
\hline 10th w & $44(22.7 \%)$ & $<0.001$ & $103(53.1 \%)$ & $<0.001$ & $<0.001$ \\
\hline 11 th w & $129(66.5 \%)$ & $<0.001$ & $173(89.2 \%)$ & $<0.001$ & $<0.001$ \\
\hline 12 th w & $140(72.2 \%)$ & $<0.001$ & $178(91.8 \%)$ & 0.007 & $<0.001$ \\
\hline 13th w & $177(91.2 \%)$ & $<0.001$ & $187(96.4 \%)$ & 0.092 & 0.035 \\
\hline 18th w & $189(97.4 \%)$ & - & $192(99.0 \%)$ & - & 0.252 \\
\hline \multicolumn{6}{|c|}{ Aortic arch } \\
\hline 10th w & $54(27.8 \%)$ & $<0.001$ & $120(61.9 \%)$ & $<0.001$ & $<0.001$ \\
\hline 11th w & $124(63.9 \%)$ & $<0.001$ & $160(82.5 \%)$ & $<0.001$ & $<0.001$ \\
\hline 12th w & $142(73.2 \%)$ & $<0.001$ & $182(93.8 \%)$ & 0.041 & $<0.001$ \\
\hline 13th w & 179 (92.3\%) & $<0.001$ & 187 (96.4\%) & 0.358 & 0.079 \\
\hline 18th w & 189 (97.4\%) & - & $190(97.9 \%)$ & - & 0.735 \\
\hline \multicolumn{6}{|c|}{ Ductal arch } \\
\hline 10th w & $54(27.8 \%)$ & $<0.001$ & $120(61.9 \%)$ & $<0.001$ & $<0.001$ \\
\hline 11th w & $127(65.5 \%)$ & $<0.001$ & 167 (86.1\%) & $<0.001$ & $<0.001$ \\
\hline 12th w & $151(77.8 \%)$ & $<0.001$ & 185 (95.4\%) & 0.032 & $<0.001$ \\
\hline 13th w & $182(93.8 \%)$ & $<0.001$ & $186(95.9 \%)$ & 0.055 & 0.358 \\
\hline 18th w & 190 (97.9\%) & - & 192 (99.0\%) & - & 0.410 \\
\hline
\end{tabular}

Comparison done using chi-square test

Bold indicates significant

$\neq p$ value in comparison between 2D and color Doppler

${ }^{*} p$ value in comparison with gestational age at 18 th week

after 11 gestational weeks [9]. On the other hand, in a smaller percentage of gestations, cardiac sonographic evaluation could be possible from 10th gestational week as sonographic researchers were in harmony with the current research study findings which have revealed that the real challenge is in the pulmonary vein evaluation in early fetal echocardiographic performance, with less than $50 \%$ successfully assessed by color Doppler even at 13 gestational weeks $[10,12]$. 


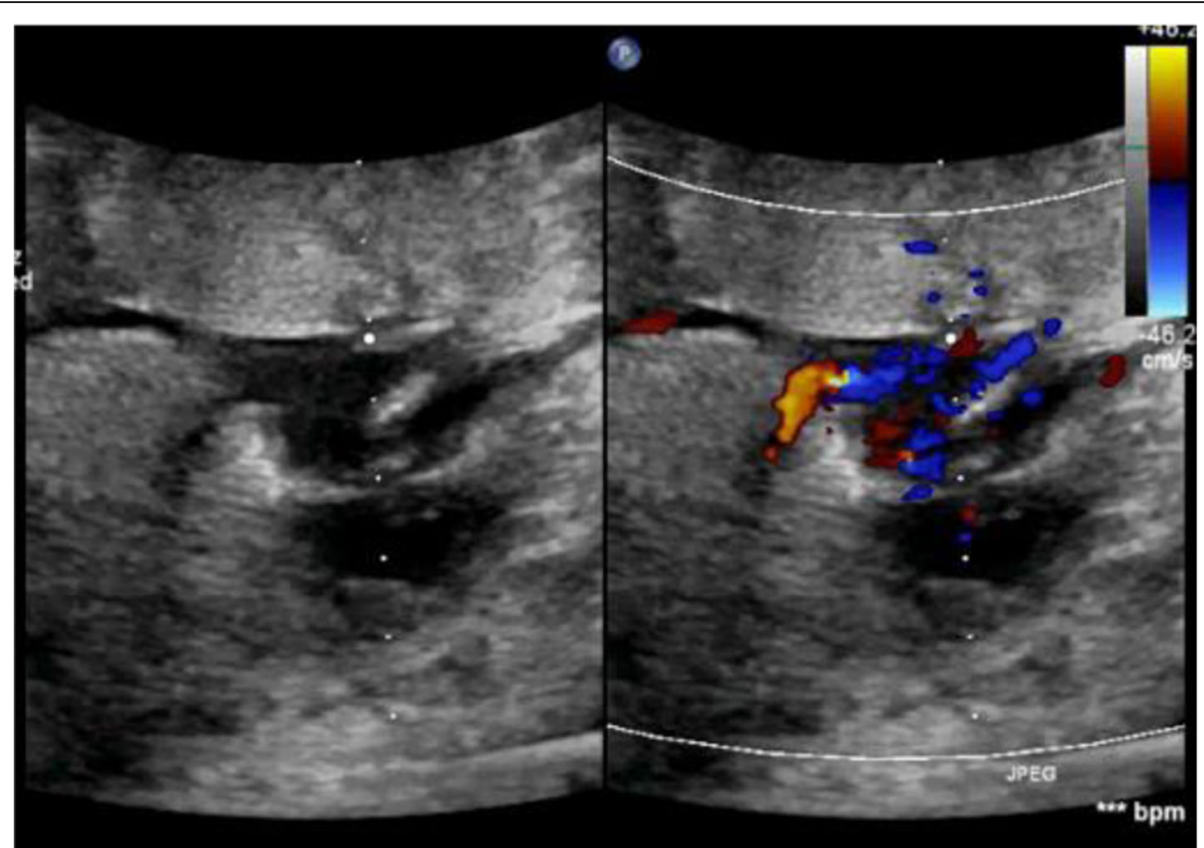

Fig. 3 Normal systemic veno-atrial connection by both 2D and color Doppler imaging in a case 12-week gestation

Developmental changes in the morphology and function of the early heart need to be considered in early fetal echocardiographic performance. Since prior research groups in a similar approach and methodology have mentioned that at 10th gestational weeks, the cardiothoracic ratio is generous, the fetal cardiac axis nearly midline, with relatively large atrial chambers in comparison to the remaining cardiac mass, and a pericardial effusion is a frequent, findings interestingly research investigators have mentioned among their findings that assessment of atrioventricular valves is problematic at earlier

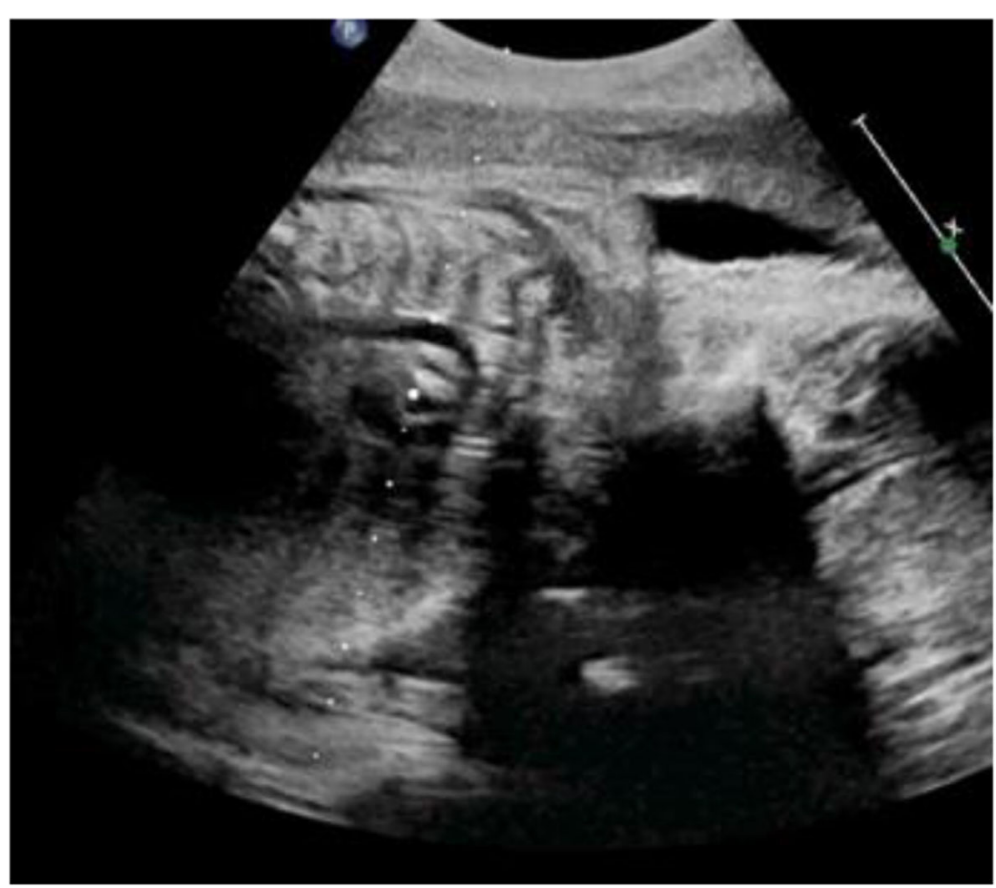

Fig. 4 2D imaging of the aortic arch in a case 13-week gestation 
gestational ages, especially before 12 gestational weeks. Those research findings could be justified by the fact that their thin anatomical nature besides the rapid fetal heart rate at earlier phases of intrauterine development impedes their proper sonographic resolution $[8,11]$.

\section{Conclusions}

First trimester fetal echocardiography is an outstanding enhancement in management pathways of cases susceptible to have fetal cardiac problems permitting early detectability of structural cardiac anomalies triggering a cascade of scanning for extra cardiac anomalies to aid in evaluation and assessment and the best management course plan for those affected cases. Yet still second trimester scanning is the gold standard, and early screening is mainly for more understanding of the development, academic, and other many purposes. It supplies cornerstone data that could be implemented in fetal progress of cardiac pathology with available observations to discuss with the parents. However, more research efforts are required in a multicentric fashion putting in consideration racial, ethnic differences in rate of fetal cardiac development besides the need to consider medical disorders such as DM that could raise the risk of cardiac anomalies in order to have in the future a clinical algorithm that could upgrade the course of management of those category of cases.

\section{Acknowledgements}

Not applicable

\section{Authors' contributions}

The authors listed below have contributed significantly to the submitted work: "Hk": Has made substantial contributions to the conception and design of the work, performing the echocardiographic analysis, and interpretation of the data and drafting of the work. "AY": Has made substantial contributions to conception and design of the work, performing the statistical analysis, interpretation of data, and substantively revised the work. All authors have read and approved the manuscript.

\section{Funding}

The authors report no financial relationships. This study received no financial support.

\section{Availability of data and materials}

The datasets generated during and/or analyzed during the current study are available from the corresponding author on reasonable request.

\section{Ethics approval and consent to participate}

Ain Shams University Institutional Ethical Committee approved the study and approved to obtain a verbal informed consent (instead of written one) from all study participants. (The committee's reference number is not available.)

\section{Author details}

'Congenital and Structural Heart Disease Unit, Department of Cardiology, Faculty of Medicine, Ain Shams University Hospital, Abbassya, Cairo, Egypt. ${ }^{2}$ Department of Obstetrics and Gynecology, Faculty of Medicine, Ain Shams University, Cairo, Egypt.

Received: 29 June 2019 Accepted: 20 March 2020

Published online: 07 April 2020

\section{References}

1. Mathews MS, MacDorman MF (2011) Infant mortality statistics from the 2007 period linked birth/infant death data set. Natl Vital Stat Rep 59:1-30

2. Levey A, Glickstein JS, Kleinman CS et al (2010) The impact of prenatal diagnosis of complex congenital heart disease on neonatal outcomes. Pediatr Cardiol 31:587-597

3. Sholler GF, Kasparian NA, Pye VE et al (2011) Fetal and post-natal diagnosis of major congenital heart disease: implications for medical and psychological care in the current era. J Paediatr Child Health 47:717-722

4. Moon-Grady A, Shahanavaz S, Brook M et al (2012) Can a complete fetal echocardiogram be performed at 12 to 16 weeks' gestation? J Am Soc Echocardiogr 25:1342-1352

5. Donofrio MT, Moon-Grady AJ, Hornberger LK et al (2014) Diagnosis and treatment of fetal cardiac disease: a scientific statement from the American Heart Association. Circulation 129:2183-2242

6. McBrien A, Howley L, Yamamoto $Y$ et al (2013) Changes in the cardiac axis from 8 to $14+6$ weeks of gestation. Ultrasound Obstet Gynecol 42:653-658

7. Bhat AH, Kehl DW, Tacy TA et al (2013) Diagnosis of tetralogy of Fallot and its variants in the late first and early second trimester: details of initial assessment and comparison with later fetal diagnosis. Echocardiography 30:81-87

8. Nemescu D, Onofriescu M (2015) Factors affecting the feasibility of routine first trimester fetal echocardiography. J Ultrasound Med 34:161-166

9. Hutchinson D, McBrien A, Howley L et al (2017) First-trimester fetal echocardiography: Identification of cardiac structures for screening from 6 to 13 weeks' gestational age. J Am Soc Echocardiogr 30(8):763-772

10. Hiescu D, Tudorache S, Comanescu A et al (2013) Improved detection rate of structural abnormalities in the first trimester using an extended examination protocol. Ultrasound Obstet Gynecol 42(3):300-309

11. Jicinska $H$, Vlasin $P$, Jicinsky $M$ et al (2017) Does first-trimester screening modify the natural history of congenital heart disease? Analysis of outcome of regional cardiac screening at 2 different time periods. Circulation 135(11): 1045-1055

12. Nemescu D, Berescu A (2015) Acoustic output measured by thermal and mechanical indices during fetal echocardiography at the time of the first trimester scan. Ultrasound Med Biol 41(1):35-39

13. Nemescu D, Onofriescu M (2015) Factors affecting the feasibility of routine first-trimester fetal echocardiography. J Ultrasound Med 34(1):161-166

14. Weiner Z, Lorber A, Shalev E (2002) Diagnosis of congenital heart defects between 11- and 14-weeks' gestation in high risk patients. J Ultrasound Med 21:23-29

15. Yu R, Li SL, Luo GY et al (2016) First-trimester echocardiographic features and perinatal outcomes in fetuses with congenital absence of the aortic valve. J Ultrasound Med 35(4):739-745

16. Zidere V, Bellsham-Revell H, Persico N et al (2013) Comparison of echocardiographic findings in fetuses at less than 15 weeks' gestation with later cardiac evaluation. Ultrasound Obstet Gynecol 42(6):679-686

\section{Publisher's Note}

Springer Nature remains neutral with regard to jurisdictional claims in published maps and institutional affiliations.

\section{Consent for publication}

Not applicable

\section{Competing interests}

The authors declare that they have no competing interests. 